ESAIM: PROCEEDINGS, July 2007, Vol.18, 1-10

Jean-Frédéric Gerbeau \& Stéphane Labbé, Editors

\title{
DISCRETE BESOV FRAMEWORK FOR FINITE VOLUME APPROXIMATION OF THE $p$-LAPLACIAN ON NON-UNIFORM CARTESIAN GRIDS
}

\author{
Boris Andreianov ${ }^{1}$, Franck Boyer $^{2}$ And Florence Hubert ${ }^{2}$
}

\begin{abstract}
This work addresses the problem of a priori error analysis for the finite volume approximation of solutions of the $p$-laplacian on cartesian grids. We first recall the way we constructed such schemes and the different error bounds we proved in our previous works. Then we concentrate particularly on the case where the exact solution has only weak regularity properties (which are natural for this problem) of Besov kind with derivation index in between 1 and 2. In this framework, the usual techniques to obtain error estimates for finite volumes schemes are not straightfoward to apply. Hence, we propose to take advantage of the variational structure of the equation and the schemes in order to obtain the error bounds. In the case of uniform meshes, this strategy was succesfully applied in [2] to obtain optimal estimates. We propose in this work to extend this approach to a more general family of quasi-uniform meshes.
\end{abstract}

Résumé. Ce travail concerne l'analyse d'erreur a priori pour l'approximation par une méthode de volumes finis des solutions du p-laplacien sur un maillage cartésien. Nous commençons par rappeller la construction de tels schémas et les différents résultats obtenus dans nos travaux antérieurs. On s'intéresse ensuite à l'analyse d'erreur dans le cas où la solution exacte possède seulement des propriétés de régularité assez faibles (naturelles pour le problème étudié) de type Besov d'indice de dérivation strictement compris entre 1 et 2. Dans ce cadre, les méthodes usuelles d'estimation d'erreur sont difficilement applicables. On propose donc de profiter de la structure variationnelle de l'équation et des schémas pour obtenir les bornes souhaitées. Sous la restriction de l'uniformité des maillages, cette approche a été utilisée avec succès dans [2] pour obtenir des estimations optimales. Nous présentons, dans ce travail, l'extension de la méthode à une famille plus générale de maillages quasi-uniformes.

\section{INTRODUCTION}

In this article we are interested in the finite volume numerical approximation on rectangular meshes of solutions to the p-laplacian with homogeneous Dirichlet boundary conditions $(1<p<+\infty)$ :

$$
-\operatorname{div}\left(|\nabla \bar{u}|^{p-2} \nabla \bar{u}\right)=f, \quad \text { in } \Omega, \quad \bar{u}=0, \quad \text { on } \partial \Omega,
$$

where the rectangular domain is $\Omega=\left[0, L_{x}\right] \times\left[0, L_{y}\right]$. Assume $L_{x}=L_{y}=1$ for simplicity. Even though the problem above is well-posed for any $f \in W^{-1, p^{\prime}}(\Omega), p^{\prime}=\frac{p}{p-1}$, we are interested here in the case where $f \in L^{p^{\prime}}(\Omega)$. In some previous works [1-3], we carried out the error analysis for a family of "variational" finite

\footnotetext{
1 Département de Mathématiques, Université de Franche-Comté, 16 route de Gray, 25030 Besançon Cedex, France

e-mail: borisa@math.univ-fcomte.fr

2 Laboratoire d'Analyse, Topologie et Probabilités, 39 rue F. Joliot Curie, 13453 Marseille Cedex 13, France

e-mail: fboyer@cmi.univ-mrs.fr \& fhubert@cmi.univ-mrs.fr 
volume schemes on cartesian meshes for this problem. The cartesian geometry of the grids we consider is of course restrictive but has the advantage to lead to relatively simple nine-points schemes, for which much deeper analysis can be performed. Moreover, using a fictitious domain and penalization approach, it is possible to reduce the resolution of (1) on any domain to cartesian geometries (see [1] for some details). Finally, we refer to $[4,7]$ for the study of finite volume schemes for such nonlinear monotonic elliptic equations on much more general grids than cartesian ones. The error analysis available for these schemes cover the case of $W^{2, p}$ solutions but, to our knowledge, the case of Besov solutions is far from being understood in this more general setting.

In [1], assuming the $W^{2, p}$ regularity of the exact solution $\bar{u}$ of (1), we derive estimates of order $p-1$ for $p \in(1,2]$, and of order $1 /(p-1)$ for $p \geq 2$ for the schemes under study in this paper; these estimates are in discrete $W^{1, p}$ norm. In [3], assuming the $W^{4,1}$ regularity of $\bar{u}$ and the uniformity of the meshes, we improve these estimates (we get in particular the $2 /(p-1)$ convergence rate for $p \geq 4$ ) and also derive $L^{\infty}$ and $W^{1,1}$ superconvergence estimates that are sharp in some cases.

Note that in the case $p \in(1,2]$, the $W^{2,2}$ regularity holds true whenever $f \in L^{p^{\prime}}(\Omega)$. The situation for $p>2$ is quite different. The following Besov regularity result (the definition and description of Besov spaces are recalled in the sequel) due to Simon [10] is essentially sharp:

Theorem 1.1. For any $p>2$ and $f \in L^{p^{\prime}}(\Omega)$, the unique solution to $(1)$ lies in the Besov space $B_{\infty}^{1+\frac{1}{p-1}, p}(\Omega)$.

Moreover, to our knowledge no regularity assumption on $f$ is known to imply $W^{2, p}$ regularity of the solution $\bar{u}$ for $p>2$ (except for the particular case of solutions without critical points; see the discussion in [3]).

Inspired by the "natural" regularity result of Theorem 1.1, we introduced in [2] the "discrete Besov" framework for uniform cartesian meshes and carried out the corresponding error analysis: the convergence rate of $\frac{2}{p(p-1)}$ was obtained. Note that the numerical resultats provided in [2] suggest that this rate is optimal for solutions in $B_{\infty}^{1+\frac{1}{p-1}, p}(\Omega)$.

The sharp technique used for the error analysis in [2] is adapted from the work [6] (based on the original idea in [12]), concerning the improvement of the error bound for $P^{1}$ finite element approximation of $W^{2, p}$ solutions. Note that the adaptation of the "finite element" technique of $[6,12]$ to the finite volume framework is not straightforward, and makes it necessary to work in the natural regularity space $B_{\infty}^{1+\frac{1}{p-1}, p}$ instead of $W^{2, p}$ (see [2, Remark 3.2]).

The goal of the present paper is to extend the discrete Besov error analysis to some non-uniform cartesian meshes. The idea is to use the characterization of Besov spaces by non uniform "translation operators", that is flows along $\mathcal{C}^{2}$ vector fields. This induces a restriction on the geometry of the mesh (see (2),(3) below) which means that the mesh parameters change in a smooth $\mathcal{C}^{2}$ way from one cell to any of its neighbors.

Although the present ideas can be applicable for very particular non quasi-uniform cartesian meshes, the problem of extending the discrete Besov analysis to non regular (especially non cartesian) meshes seems to be open and quite challenging.

Note that the results of this paper extend in a straightforward way to more general nonlinear elliptic equations arising from the minimization of a functional defined on $W_{0}^{1, p}(\Omega)$ of the form $J: u \mapsto \int_{\Omega} \Phi(\nabla u)-f u$, where $\Phi$ should be assumed strictly convex, coercive and such that $\nabla \Phi$ enjoying the same growth restrictions and (local) Hölder continuity properties as $z \mapsto|z|^{p-2} z$.

The outline of this paper is the following. In section 2, we describe the meshes we are interested in, we also introduce some notations and we finally recall the definition of the finite volume schemes under study. In section 3, we state the main result of this paper (Theorem 3.1) which provides an error bound for Besov solutions of the $p$-laplacian then we recall the main steps of the proof (following [2]). Finally in section 4 , we give the proofs of Propositions 3.3 and 3.4 in the case of the quasi-uniform meshes under study. 


\section{The finite volume scheme}

\subsection{Definition of the meshes}

We consider in this paper families of rectangular meshes defined on the domain $\Omega$. They are assumed to be belong the class $\mathfrak{C}$ defined as follows: we suppose given two increasing $\mathcal{C}^{2}$ diffeomorphisms from $\mathbb{R}$ onto itself denoted $g_{\mathrm{x}}, g_{\mathrm{y}}$ and such that $g_{\mathrm{x}}(0)=g_{\mathrm{y}}(0)=0, g_{\mathrm{x}}(1)=g_{\mathrm{y}}(1)=1$. We denote by $g=g_{\mathrm{x}} \otimes g_{\mathrm{y}}$ the map defined by $g(x, y)=\left(g_{\mathrm{x}}(x), g_{\mathrm{y}}(y)\right)$ and by $\theta_{\mathrm{x}}, \theta_{\mathrm{y}}$ the two vector fields given by $\theta_{\mathrm{x}}(x, y)=1 / 2\left(g_{\mathrm{x}}^{\prime}\left(g_{\mathrm{x}}^{-1}(x)\right), 0\right)$ and $\theta_{\mathrm{y}}(x, y)=1 / 2\left(0, g_{\mathrm{y}}^{\prime}\left(g_{\mathrm{y}}^{-1}(y)\right)\right)$.

For any two integers $N_{x}$ and $N_{y}$, we let $h=\frac{1}{N_{x}}, k=\frac{1}{N_{y}}$ and we construct the mesh $\mathcal{T}$ on $\Omega$ as the image of the uniform rectangular $N_{x} \times N_{y}$ grid on $[0,1]^{2}$ through the map $g$. In this setting, the control volumes $\mathcal{K}_{i j}$ and their centers $x_{\mathcal{K}_{i j}}$ (which are not the geometric centers of the control volumes) are defined by

$$
\left.\mathcal{K}_{i j}=\right] g_{\mathrm{x}}((i-1) h), g_{\mathrm{x}}(i h)[\times] g_{\mathrm{y}}((j-1) k), g_{\mathrm{y}}(j k)\left[\text {, and } x_{\mathcal{K}_{i j}}=\left(g_{\mathrm{x}}((i-1 / 2) h), g_{\mathrm{y}}((j-1 / 2) k)\right)\right. \text {, }
$$

for any $1 \leq i \leq N_{x}$ and $1 \leq j \leq N_{y}$. In order to measure the regularity of this family of meshes, we introduce

$$
\operatorname{reg}(\mathcal{T})=\max \left(\frac{h}{k}, \frac{k}{h},\left\|g^{\prime}\right\|_{\infty}+\left\|g^{\prime \prime}\right\|_{\infty},\left\|\left(g^{-1}\right)^{\prime}\right\|_{\infty}\right),
$$

finally the size of the mesh is defined by $\operatorname{size}(\mathcal{T})=\max _{i j} \operatorname{diam}\left(\mathcal{K}_{i j}\right)$.

As in $[1,2]$, we introduce artificial control volumes outside the domain obtained by symmetry with respect to the boundaries of $\Omega$ in order to treat the boundary conditions. Now we can define the dual control volumes $\mathcal{K}^{*}$ as the rectangles whose vertices are the points $x_{\mathcal{K}}$ of four adjacent control volumes (see Figure 1).
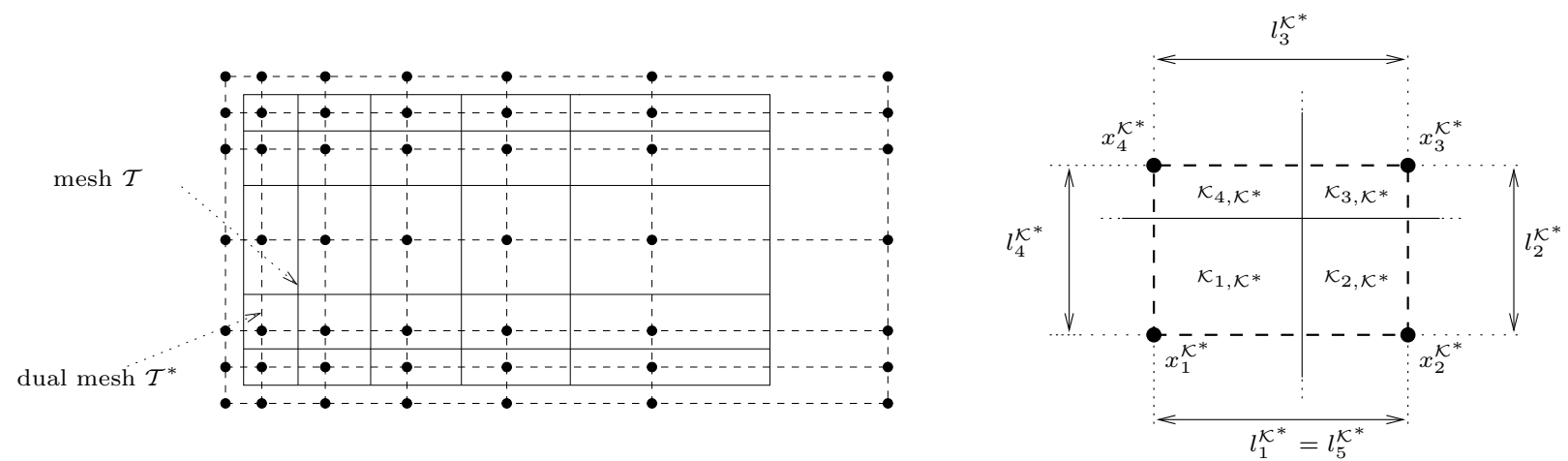

FiguRE 1. Non-uniform mesh (left) and notations in a dual control volume (right)

For any dual control volume $\mathcal{K}^{*}$ (see Figure 1$),\left(x_{i}^{\mathcal{K}^{*}}\right)_{i=1,2,3,4}$ are the vertices of the dual control volume $\mathcal{K}^{*}$ numbered counter clockwise starting from the lower left hand corner; $\left(\mathcal{K}_{i}^{\mathcal{K}^{*}}\right)_{i=1,2,3,4}$ are the corresponding control volumes with centers $\left(x_{i}^{\mathcal{K}^{*}}\right)_{i=1,2,3,4} ; \sigma_{i}^{\mathcal{K}^{*}}$ is the half-edge between $\mathcal{K}_{i}^{\mathcal{K}^{*}}$ and $\mathcal{K}_{i+1}^{\mathcal{K}^{*}}$ located in $\mathcal{K}^{*}$ and $m_{i}^{\mathcal{K}^{*}}$ denotes its measure; $l_{i}^{\mathcal{K}^{*}}$ is the distance between $x_{i}^{\mathcal{K}^{*}}$ and $x_{i+1}^{\mathcal{K}^{*}}$; finally, $\tau_{i}^{\mathcal{K}^{*}}=\frac{m_{i}^{\mathcal{K}^{*}}}{l_{i}^{\mathcal{K}^{*}}}$ is the so-called transmissivity coefficient. Conventionally, in a given dual control volume, the indexes $i \in \mathbb{Z}$ are understood modulo 4 .

The finite volume method associates to each control volume $\mathcal{K}$ an unknown value $u_{\mathcal{K}}$. We denote the set $\left(u_{\mathcal{K}}\right)_{\mathcal{K} \in \mathcal{T}} \in \mathbb{R}^{\mathcal{T}}$ by $u^{\mathcal{T}}$. For any continuous function $v$ on $\Omega$, the discrete function $v^{\mathcal{T}}=\left(v_{\mathcal{K}}\right)_{\mathcal{K} \in \mathcal{T}}$ with $v_{\mathcal{K}}=v\left(x_{\mathcal{K}}\right)$, is called the projection of $v$ on the space $\mathbb{R}^{\mathcal{T}}$ of discrete functions. For a given discrete function $u^{\mathcal{T}} \in \mathbb{R}^{\mathcal{T}}$, the homogeneous Dirichlet boundary conditions are taken into account by using the ghost cells method. That is, we extend the values of $u^{\mathcal{T}}$ on artificial points outside of $\Omega$ by odd symmetry with respect to the corresponding boundaries (see the details in $[1,2]$ ). 
Given a dual control volume $\mathcal{K}^{*}$, we define the projection operator $T_{\mathcal{K}^{*}}$ which associates to each $u^{\mathcal{T}} \in \mathbb{R}^{\mathcal{T}}$ its values $T_{\mathcal{K}^{*}}\left(u^{\mathcal{T}}\right)=\left(u_{1, \mathcal{K}^{*}}^{\mathcal{T}}, u_{2, \mathcal{K}^{*}}^{\mathcal{T}}, u_{3, \mathcal{K}^{*}}^{\mathcal{T}}, u_{4, \mathcal{K}^{*}}^{\mathcal{T}}\right)$ in the four control volumes $\left(\mathcal{K}_{i}^{\mathcal{K}^{*}}\right)_{i}$ that intersect $\mathcal{K}^{*}$. Note that for boundary dual control volumes, ghost cells are used in order to give sense to the definition of $T_{\mathcal{K}^{*}}$. For instance, if $\mathcal{K}^{*}$ is located at the right boundary of $\Omega$, we have by definition $u_{2, \mathcal{K}^{*}}^{\mathcal{T}}=-u_{1, \mathcal{K}^{*}}^{\mathcal{T}}$ and $u_{3, \mathcal{K}^{*}}^{\mathcal{T}}=-u_{4, \mathcal{K}^{*}}^{\mathcal{T}}$.

\subsection{Discrete Lebesgue, Sobolev and Besov norms}

Denote by $\mathbf{1}_{\mathcal{K}}$ the characteristic function of the control volume $\mathcal{K}$. Each discrete function $u^{\mathcal{T}} \in \mathbb{R}^{\mathcal{T}}$ is identified with the bounded function $u^{\mathcal{T}}=\sum_{\mathcal{K} \in \mathcal{T}} u_{\mathcal{K}} \mathbf{1}_{\mathcal{K}}$, so that for $r \in[1,+\infty]$ the norms $\left\|u^{\mathcal{T}}\right\|_{L^{r}}$ are naturally defined. For any $u^{\mathcal{T}} \in \mathbb{R}^{\mathcal{T}}$, and any $\mathcal{K}^{*} \in \mathcal{T}^{*}$, set

$$
\delta_{i}^{\mathcal{K}^{*}}\left(u^{\mathcal{T}}\right)=\frac{u_{i+1, \mathcal{K}^{*}}^{\mathcal{T}}-u_{i, \mathcal{K}^{*}}^{\mathcal{T}}}{l_{i}^{\mathcal{K}^{*}}}, \quad i \in\{1, \ldots, 4\}, \quad \text { and }\left|u^{\mathcal{T}}\right|_{1, \mathcal{K}^{*}}=\left(\frac{1}{2} \sum_{i=1}^{4}\left|\delta_{i}^{\mathcal{K}^{*}}\left(u^{\mathcal{T}}\right)\right|^{2}\right)^{\frac{1}{2}}
$$

Then, we define the discrete $W_{0}^{1, p}$ norm of any $u^{\mathcal{T}} \in \mathbb{R}^{\mathcal{T}}$ by

$$
\left\|u^{\mathcal{T}}\right\|_{1, p, \mathcal{T}}=\left(\sum_{\mathcal{K}^{*} \in \mathcal{T}^{*}} m\left(\mathcal{K}^{*} \cap \Omega\right)\left|u^{\mathcal{T}}\right|_{1, \mathcal{K}^{*}}^{p}\right)^{\frac{1}{p}} .
$$

The following Poincaré inequality holds in this discrete setting (see [1, Lemma 2.4] and [5]):

$$
\left\|u^{\mathcal{T}}\right\|_{L^{p}} \leq C(p) \operatorname{diam}(\Omega)\left\|u^{\mathcal{T}}\right\|_{1, p, \mathcal{T}}, \quad \forall u^{\mathcal{T}} \in \mathbb{R}^{\mathcal{T}} .
$$

For any $0<\alpha<1$, we define the discrete $B_{\infty}^{1+\alpha, p}$ norm of $u^{\mathcal{T}} \in \mathbb{R}^{\mathcal{T}}$ by

$$
\left\|u^{\mathcal{T}}\right\|_{1+\alpha, p, \mathcal{T}}=\left\|u^{\mathcal{T}}\right\|_{1, p, \mathcal{T}}+\left(\sum_{\mathcal{K}^{*} \in \mathcal{T}^{*}} m\left(\mathcal{K}^{*} \cap \Omega\right)\left|\frac{\delta_{1}^{\mathcal{K}^{*}}\left(u^{\mathcal{T}}\right)+\delta_{3}^{\mathcal{K}^{*}}\left(u^{\mathcal{T}}\right)}{\left(l_{2, \mathcal{K}^{*}}\right)^{\alpha}}\right|^{p}\right)^{\frac{1}{p}} .
$$

Let us comment on the definition (5). The Besov space $B_{\infty}^{1+\alpha, p}(\Omega)$ can be defined, for instance, as the real interpolation space $\left(W^{1, p}(\Omega), W^{2, p}(\Omega)\right)_{\alpha, \infty}$ by the $K$-method of Peetre (see [8,11]). It can also be characterized by means of translation-like operators as follows. For any vector field $\theta \in \mathcal{C}^{2}\left(\mathbb{R}^{2}, \mathbb{R}^{2}\right)$ we denote by $e^{s \theta}$ its corresponding flow defined by

$$
e^{0 \theta}=\mathrm{Id}, \frac{d}{d s} e^{s \theta}(z)=\theta\left(e^{s \theta}(z)\right), \forall z \in \mathbb{R}^{2} .
$$

We also introduce, for any $s>0$, the set $\Omega_{\theta}^{s}=\left\{z \in \Omega, \quad\left(e^{r \theta}(z)\right)_{r \in[0, s]} \subset \Omega\right\}$. Finally, for any $v \in W^{1, p}(\Omega)$ and $\alpha \in] 0,1\left[\right.$, we set $\|v\|_{\theta, B_{\infty}^{1+\alpha, p}}=\sup _{s>0}\left(\int_{\Omega_{\theta}^{s}}\left|\frac{\nabla v \circ e^{s \theta}(z)-\nabla v(z)}{s^{\alpha}}\right|^{p} d z\right)^{\frac{1}{p}}$. Then, $v \in W^{1, p}(\Omega)$ belongs to $B_{\infty}^{1+\alpha, p}(\Omega)$ iff $\|v\|_{\theta, B_{\infty}^{1+\alpha, p}}<+\infty$ for all $\theta \in \mathcal{C}^{2}\left(\mathbb{R}^{2}, \mathbb{R}^{2}\right)$.

In the same spirit, the quantity $\left\|u^{\mathcal{T}}\right\|_{1+\alpha, p, \mathcal{T}}$ contains translation information on $u^{\mathcal{T}}$ that encodes its discrete Besov regularity. Proposition 3.3 below links the discrete and continuous Besov semi-norms.

\subsection{Description of the finite volume approximation}

The general form of symmetric, locally conservative finite volume schemes (with reasonable stencil) on cartesian meshes that are consistent with piecewise affine functions was described in [1]. It was assumed that $x_{\mathcal{K}}$ is the geometrical center of the control volume $x_{\mathcal{K}}$ but this is in fact unnecessary (as soon as the dual control 
volumes are still rectangular) and the properties of the schemes still hold. These schemes can be written as the following system of nonlinear equations:

$$
a\left(u^{\mathcal{T}}\right) \stackrel{\text { def }}{=} \sum_{\mathcal{K}^{*} \in \mathcal{T}^{*}} m\left(\mathcal{K}^{*} \cap \Omega\right) T_{\mathcal{K}^{*}}^{t} \circ a_{\mathcal{K}^{*}} \circ T_{\mathcal{K}^{*}}\left(u^{\mathcal{T}}\right)=\left(m(\mathcal{K}) f_{\mathcal{K}}\right)_{\mathcal{K} \in \mathcal{T}}
$$

where $u^{\mathcal{T}} \in \mathbb{R}^{\mathcal{T}}$ is the discrete function called approximate solution and $f_{\mathcal{K}}$ the mean value of the function $f$ on each control volume $\mathcal{K}$. Furthermore, for each dual control volume $\mathcal{K}^{*}$, the maps $a_{\mathcal{K}^{*}}: \mathbb{R}^{4} \rightarrow \mathbb{R}^{4}$ acting on the four values $\left(u_{i, \mathcal{K}^{*}}^{\mathcal{T}}\right)_{\{i=1,2,3,4\}}$ are defined by:

$$
a_{\mathcal{K}^{*}}(v) \stackrel{\text { def }}{=}\left(B_{\mathcal{K}^{*}} v, v\right)^{\frac{p-2}{2}} B_{\mathcal{K}^{*}} v, \quad \forall v \in \mathbb{R}^{4},
$$

where $B_{\mathcal{K}^{*}}$ is a $4 \times 4$ symmetric matrix defined by the choice of one parameter $\xi^{\mathcal{K}^{*}}$ as follows:

$$
B_{\mathcal{K}^{*}}=\frac{1}{m\left(\mathcal{K}^{*}\right)}\left(\begin{array}{cccc}
2 \xi^{\mathcal{K}^{*}}+\tau_{1}^{\mathcal{K}^{*}}+\tau_{4}^{\mathcal{K}^{*}} & -2 \xi^{\mathcal{K}^{*}}-\tau_{1}^{\mathcal{K}^{*}} & 2 \xi^{\mathcal{K}^{*}} & -2 \xi^{\mathcal{K}^{*}}-\tau_{4}^{\mathcal{K}^{*}} \\
-2 \xi^{\mathcal{K}^{*}}-\tau_{1}^{\mathcal{K}^{*}} & 2 \xi^{\mathcal{K}^{*}}+\tau_{1}^{\mathcal{K}^{*}}+\tau_{2}^{\mathcal{K}^{*}} & -2 \xi^{\mathcal{K}^{*}}-\tau_{2}^{\mathcal{K}^{*}} & 2 \xi^{\mathcal{K}^{*}} \\
2 \xi^{\mathcal{K}^{*}} & -2 \xi^{\mathcal{K}^{*}}-\tau_{2}^{\mathcal{K}^{*}} & 2 \xi^{\mathcal{K}^{*}}+\tau_{2}^{\mathcal{K}^{*}}+\tau_{3}^{\mathcal{K}^{*}} & -2 \xi^{\mathcal{K}^{*}}-\tau_{3}^{\mathcal{K}^{*}} \\
-2 \xi^{\mathcal{K}^{*}}-\tau_{4}^{\mathcal{K}^{*}} & 2 \xi^{\mathcal{K}^{*}} & -2 \xi^{\mathcal{K}^{*}}-\tau_{3}^{\mathcal{K}^{*}} & 2 \xi^{\mathcal{K}^{*}}+\tau_{3}^{\mathcal{K}^{*}}+\tau_{4}^{\mathcal{K}^{*}}
\end{array}\right),
$$

so that $\left(B_{\mathcal{K}^{*}} v, v\right)^{1 / 2}$ is an approximation of the norm of the gradient of the solution in the dual control volume $\mathcal{K}^{*}$. Such a finite volume scheme on the mesh $\mathcal{T}$ is then completely characterized by the family of parameters $\Xi=\left(\xi^{\mathcal{K}^{*}}\right)_{\mathcal{K}^{*} \in \mathcal{T}^{*}}$. In order for the scheme to be well-defined we need that $2 \xi^{\mathcal{K}^{*}}+\frac{m_{1}^{\mathcal{K}^{*}} m_{3}^{\mathcal{K}^{*}}+m_{2}^{\mathcal{K}^{*}} m_{4}^{\mathcal{K}^{*}}}{l_{1}^{\mathcal{K}^{*}} l_{2}^{\mathcal{K}^{*}}}>0$, in which case $B_{\mathcal{K}^{*}}$ is positive with rank 3 .

As shown in [1], (7)-(8) ensure the consistency and the symmetry of the scheme. In addition to $\operatorname{reg}(\mathcal{T})$ in (3), we define two quantities measuring regularity of the meshes and the schemes.

Definition 2.1. For a given mesh $\mathcal{T}$ and a set of parameters $\Xi=\left(\xi^{\mathcal{K}^{*}}\right)_{\mathcal{K}^{*} \in \mathcal{T}^{*} \text {, set }}$

$\operatorname{adm}(\mathcal{T}, \Xi)=\max \left(\max _{\mathcal{K}^{*} \in \mathcal{T}^{*}} \xi^{\mathcal{K}^{*}},\left(\min _{\mathcal{K}^{*} \in \mathcal{T}^{*}}\left(2 \xi^{\mathcal{K}^{*}}+\frac{m_{1}^{\mathcal{K}^{*}} m_{3}^{\mathcal{K}^{*}}+m_{2}^{\mathcal{K}^{*}} m_{4}^{\mathcal{K}^{*}}}{l_{1}^{\mathcal{K}^{*}} l_{2}^{\mathcal{K}^{*}}}\right)\right)^{-1}\right)$, and $\operatorname{reg}(\Xi)=\frac{\max ^{*} \cap \mathcal{L}^{*} \neq \emptyset\left|\xi^{\mathcal{K}^{*}}-\xi^{\mathcal{L}^{*}}\right|}{h}$

Here and in the sequel $C$ denotes a generic constant depending only on $p, \Omega, \operatorname{reg}(\mathcal{T}), \operatorname{adm}(\mathcal{T}, \Xi) \operatorname{and} \operatorname{reg}(\Xi)$. We refer to $[1,2]$ for precise dependencies in each case. Using this convention we have (see [1, Lemma 2.8])

$$
\frac{1}{C}\left|u^{\mathcal{T}}\right|_{1, \mathcal{K}^{*}} \leq\left|B_{\mathcal{K}^{*}}^{\frac{1}{2}} T_{\mathcal{K}^{*}}\left(u^{\mathcal{T}}\right)\right| \leq C\left|u^{\mathcal{T}}\right|_{1, \mathcal{K}^{*}}, \forall \mathcal{K}^{*} \in \mathcal{T}^{*}, \forall u^{\mathcal{T}} \in \mathbb{R}^{\mathcal{T}}
$$

\subsection{Discrete energy}

We call discrete energy of the scheme the following functional $J_{\mathcal{T}}$, depending on $\Xi$, acting on discrete functions

$$
J_{\mathcal{T}}\left(v^{\mathcal{T}}\right) \stackrel{\text { def }}{=} \frac{1}{p}\left(a\left(v^{\mathcal{T}}\right), v^{\mathcal{T}}\right)-\sum_{\mathcal{K} \in \mathcal{T}} m(\mathcal{K}) f_{\mathcal{K}} v_{\mathcal{K}}=\frac{1}{p} \sum_{\mathcal{K}^{*} \in \mathcal{T}^{*}} m\left(\mathcal{K}^{*} \cap \Omega\right)\left|B_{\mathcal{K}^{*}}^{\frac{1}{2}} T_{\mathcal{K}^{*}}\left(v^{\mathcal{T}}\right)\right|^{p}-\sum_{\mathcal{K} \in \mathcal{T}} m(\mathcal{K}) f_{\mathcal{K}} v_{\mathcal{K}}, \quad \forall v^{\mathcal{T}} \in \mathbb{R}^{\mathcal{T}} .
$$

For any $p>2$, we have (see [1, Corollary 2.11])

$$
J_{\mathcal{T}}\left(v^{\mathcal{T}}\right)-J_{\mathcal{T}}\left(u^{\mathcal{T}}\right)-\left(\nabla J_{\mathcal{T}}\left(u^{\mathcal{T}}\right), v^{\mathcal{T}}-u^{\mathcal{T}}\right) \geq C\left\|u^{\mathcal{T}}-v^{\mathcal{T}}\right\|_{1, p, \mathcal{T}}^{p}, \forall u^{\mathcal{T}}, v^{\mathcal{T}} \in \mathbb{R}^{\mathcal{T}}
$$

This implies that $J_{\mathcal{T}}$ is strictly convex and coercive. It is then easy to see that its unique minimizing point $u^{\mathcal{T}}$ is the unique solution of the discrete equations (6) and satisfies in addition the estimate

$$
\left\|u^{\mathcal{T}}\right\|_{1, p, \mathcal{T}} \leq C\|f\|_{L^{p^{\prime}}}^{\frac{1}{p-1}}
$$




\section{ERror ANALYSis FOr SOLUtions With Besov REgUlarity}

We can now prove the following error estimate for the solutions to (1) for any source term $f \in L^{p^{\prime}}(\Omega)$, that is to say for any solution lying in $B_{\infty}^{1+\frac{1}{p-1}, p}(\Omega)$ (thanks to Theorem 1.1).

Theorem 3.1. Let $p>2$ and $\mathcal{T}$ be a mesh of $\Omega$ in the class $\mathfrak{C}$ of quasi-uniform meshes defined in Section 2.1 . For any $f \in L^{p^{\prime}}(\Omega)$, we have

$$
\left\|u^{\mathcal{T}}-\bar{u}^{\mathcal{T}}\right\|_{1, p, \mathcal{T}} \leq C \operatorname{size}(\mathcal{T})^{\alpha_{p}}\|f\|_{L^{p^{\prime}}}^{\frac{1}{p-1}}
$$

with $\alpha_{p}=\frac{2}{p(p-1)}$ if $p \geq 3$ and $\alpha_{p}=\frac{1}{p}$ if $2<p<3$. In this estimate $u^{\mathcal{T}}$ is the unique solution to the scheme (6) and $\bar{u}^{\mathcal{T}}$ the projection of the exact solution $\bar{u}$ of $(1)$ defined by $\bar{u}_{\mathcal{K}}=\bar{u}\left(x_{\mathcal{K}}\right)$, for any $\mathcal{K} \in \mathcal{T}$.

Just like in [2], in the case $2<p<3$, if $f$ lies in the Hölder space $\mathcal{C}^{0, \frac{3-p}{p-2}}(\bar{\Omega})$ then we can recover an error estimate in $\operatorname{size}(\mathcal{T})^{\frac{2}{p(p-1)}}$, which approaches the first order convergence when $p$ tends to 2 .

This result is formally the same than in [2] except the fact that we allow more general non-uniform meshes in the present work. The overall principle of the proof is the same than in this reference. We only recall here the main steps, the details being available in [2]. We finally give in section 4 the detailed proofs of the new elements necessary to take into account non uniform meshes.

Sketch of proof. For $v^{\mathcal{T}} \in \mathbb{R}^{\mathcal{T}}$, define by $\Pi_{\mathcal{T}} v^{\mathcal{T}}$ the piecewise affine interpolation of $v^{\mathcal{T}}$ on triangles obtained by bissection of dual control volumes (see [2] for details). Since the approximate solution $u^{\mathcal{T}}$ is the minimizer of $J_{\mathcal{T}}$ over $\mathbb{R}^{\mathcal{T}}$, we have $\nabla J_{\mathcal{T}}\left(u^{\mathcal{T}}\right)=0$, so that (10) yields $C\left\|u^{\mathcal{T}}-\bar{u}^{\mathcal{T}}\right\|_{1, p, \mathcal{T}}^{p} \leq J_{\mathcal{T}}\left(\bar{u}^{\mathcal{T}}\right)-J_{\mathcal{T}}\left(u^{\mathcal{T}}\right)$. It follows

$$
C\left\|u^{\mathcal{T}}-\bar{u}^{\mathcal{T}}\right\|_{1, p, \mathcal{T}}^{p} \leq\left[J_{\mathcal{T}}\left(\bar{u}^{\mathcal{T}}\right)-J\left(\Pi_{\mathcal{T}} \bar{u}^{\mathcal{T}}\right)\right]+\left[J\left(\Pi_{\mathcal{T}} \bar{u}^{\mathcal{T}}\right)-J(\bar{u})\right]+[J(\bar{u})-J(v)]+\left[J(v)-J_{\mathcal{T}}\left(u^{\mathcal{T}}\right)\right]
$$

for any $v \in W_{0}^{1, p}(\Omega)$. Since $\bar{u}$ minimize $J$ over $W_{0}^{1, p}(\Omega)$, the third term is non-positive. We choose now $v=\Pi_{\mathcal{T}} u^{\mathcal{T}}$ and we get

$$
C\left\|u^{\mathcal{T}}-\bar{u}^{\mathcal{T}}\right\|_{1, p, \mathcal{T}}^{p} \leq\left[J_{\mathcal{T}}\left(\bar{u}^{\mathcal{T}}\right)-J\left(\Pi_{\mathcal{T}} \bar{u}^{\mathcal{T}}\right)\right]+\left[J\left(\Pi_{\mathcal{T}} \bar{u}^{\mathcal{T}}\right)-J(\bar{u})\right]+\left[J\left(\Pi_{\mathcal{T}} u^{\mathcal{T}}\right)-J_{\mathcal{T}}\left(u^{\mathcal{T}}\right)\right]
$$

The middle term is classically estimated, using $P^{1}$-finite element interpolation results, by

$$
\left|J\left(\Pi_{\mathcal{T}} \bar{u}\right)-J(\bar{u})\right| \leq C\left\|\Pi_{\mathcal{T}} \bar{u}^{\mathcal{T}}-\bar{u}\right\|_{W^{1, p}}^{2}\left(\left\|\Pi_{\mathcal{T}} \bar{u}^{\mathcal{T}}\right\|_{W^{1, p}}+\|\bar{u}\|_{W^{1, p}}\right)^{p-2} \leq C \operatorname{size}(\mathcal{T})^{\frac{2}{p-1}}\|\bar{u}\|_{B_{\infty}^{1+\frac{1}{p-1}, p}}^{2}\|\bar{u}\|_{W^{1, p}}^{p-2}
$$

The other two terms in the right hand side in (13) are similar. One needs to control difference between $J\left(\Pi_{\mathcal{T}} \cdot\right)$ and $J_{\mathcal{T}}(\cdot)$ for both $u^{\mathcal{T}}$ and $\bar{u}^{\mathcal{T}}$. This can be done in terms of the discrete Besov norms as follows.

Proposition 3.2 (see [2, Lemma 3.3]). For any $\alpha \in] 0,1[$, we have

$$
\left|J\left(\Pi_{\mathcal{T}} v^{\mathcal{T}}\right)-J_{\mathcal{T}}\left(v^{\mathcal{T}}\right)\right| \leq C \operatorname{size}(\mathcal{T})^{2 \alpha}\left\|v^{\mathcal{T}}\right\|_{1+\alpha, p, \mathcal{T}}^{2}\left\|v^{\mathcal{T}}\right\|_{1, p, \mathcal{T}}^{p-2}+C \operatorname{size}(\mathcal{T})\|f\|_{L^{p^{\prime}}}\left\|v^{\mathcal{T}}\right\|_{1, p, \mathcal{T}}, \quad \forall v^{\mathcal{T}} \in \mathbb{R}^{\mathcal{T}}
$$

Finally, Theorem 3.1 will be proved if we manage to prove a uniform bound of the discrete Besov semi-norm of the approximate solution $u^{\mathcal{T}}$ and of the projection of the exact solution $\bar{u}^{\mathcal{T}}$, using the fact that we know that $\bar{u} \in B_{\infty}^{1+\frac{1}{p-1}, p}(\Omega)$ by Theorem 1.1.

These two uniform bounds were obtained in [2] in the case of uniform cartesian meshes and uniform scheme (that is when $\xi^{\mathcal{K}^{*}}=$ const). The proof given in this reference really relies on the uniformity of the mesh. At the present time we are not able to prove these estimates in the case of general cartesian mesh.

Nevertheless, in the framework of some quasi-uniform cartesian meshes as defined in Section 2.1, we prove in Section 4 the two following results which permit us to conclude the proof of the error estimate using (13), (14) and Proposition 3.2. 
Proposition 3.3. Let $\alpha \in] 0,1\left[\right.$. For any $v \in W_{0}^{1, p}(\Omega) \cap B_{\infty}^{1+\alpha, p}(\Omega)$, let $v^{\mathcal{T}}=\left(v\left(x_{\mathcal{K}}\right)\right)_{\mathcal{K} \in \mathcal{T}}$ be the projection of $v$ on $\mathbb{R}^{\mathcal{T}}$. We have

$$
\left\|v^{\mathcal{T}}\right\|_{1+\alpha, p, \mathcal{T}} \leq C_{\alpha}\left(\|v\|_{W^{1, p}}+\|v\|_{\theta_{\mathrm{x}}, B_{\infty}^{1+\alpha, p}}+\|v\|_{\theta_{\mathrm{y}}, B_{\infty}^{1+\alpha, p}}\right) .
$$

Proposition 3.4 (Discrete analogue of Theorem 1.1).

For any $f \in L^{p^{\prime}}(\Omega)$, the solution $u^{\mathcal{T}}$ of $(6)$ satisfies $\left\|u^{\mathcal{T}}\right\|\left\|_{1+\frac{1}{p-1}, p, \mathcal{T}} \leq C\right\| f \|_{L^{p^{\prime}}}^{\frac{1}{p-1}}$.

\section{Discrete Besov estimates}

\subsection{Discrete estimate for the projection of a function. Proof of Proposition 3.3}

The proof in the case of uniform meshes relies on sharp estimates of the $(1+\alpha)$-th order differential quotients appearing in the discrete Besov norm (5) by means of translation integral terms on the gradient of the function. More precisely, we shown the following estimate for any $v \in B_{\infty}^{1+\alpha, p}(\Omega)$

$$
\begin{aligned}
& m\left(\mathcal{K}^{*}\right)\left(\frac{1}{\left(l_{2}^{\mathcal{K}^{*}}\right)^{\alpha}}\left|\frac{v\left(x_{4}^{\mathcal{K}^{*}}\right)-v\left(x_{3}^{\mathcal{K}^{*}}\right)}{l_{1}^{\mathcal{K}^{*}}}-\frac{v\left(x_{1}^{\mathcal{K}^{*}}\right)-v\left(x_{2}^{\mathcal{K}^{*}}\right)}{l_{1}^{\mathcal{K}^{*}}}\right|\right)^{p} \\
& \quad \leq C \int_{\mathcal{K}^{*}}\left|\frac{\nabla v(z)-\nabla\left(\tau_{l_{1} \mathcal{K}^{*}, l_{2}} * v\right)(z)}{\operatorname{diam}\left(\mathcal{K}^{*}\right)^{\alpha}}\right|^{p} d z+C \int_{\mathcal{K}^{*}} \mid \frac{\nabla v(z)-\nabla\left(\left.\tau_{\left.-l_{1}^{\mathcal{K}^{*}}, l_{2}^{\mathcal{K}^{*}} v\right)(z)}\right|^{p} d z\right.}{\operatorname{diam}\left(\mathcal{K}^{*}\right)^{\alpha}}
\end{aligned}
$$

where $\tau_{a, b}$ is the translation operator $\tau_{a, b} v(\cdot, \cdot)=v(\cdot+a / 2, \cdot+b / 2)$. Unfortunately, this estimate is not directly usable in the present form for non uniform meshes since all the translation operators in the right-hand side may be different from one dual control volume to another and thus we do not find a bound of the discrete Besov semi-norm of $v^{\mathcal{T}}$ as a function of a quantity $\|v\|_{\tau, B_{\infty}^{1+\alpha, p}}$ for a unique given translation operator $\tau$.

Nevertheless, in the framework of smooth cartesian meshes as defined in the introduction, we can recover a usable estimate by performing a change of variables. More precisely, we define on $\widetilde{\Omega}=[0,1]^{2}$ the function $\tilde{v}=v \circ g$, where $g$ is defined in section 2. We can now apply (15) to $\tilde{v}$ on the dual control volume $g^{-1}\left(\mathcal{K}^{*}\right)$ of the uniform mesh on $\widetilde{\Omega}$ (whose vertices are denoted by $x_{1}, \ldots, x_{4}$ ):

$$
\begin{gathered}
m\left(\mathcal{K}^{*}\right)\left(\frac{1}{\left(l_{2}^{\mathcal{K}^{*}}\right)^{\alpha}}\left|\frac{v\left(x_{4}^{\mathcal{K}^{*}}\right)-v\left(x_{3}^{\mathcal{K}^{*}}\right)}{l_{1}^{\mathcal{K}^{*}}}-\frac{v\left(x_{1}^{\mathcal{K}^{*}}\right)-v\left(x_{2}^{\mathcal{K}^{*}}\right)}{l_{1}^{\mathcal{K}^{*}}}\right|\right)^{p} \leq C h k\left(\frac{1}{k^{\alpha}}\left|\frac{\tilde{v}\left(x_{4}\right)-\tilde{v}\left(x_{3}\right)}{h}-\frac{\tilde{v}\left(x_{1}\right)-\tilde{v}\left(x_{2}\right)}{h}\right|\right)^{p} \\
\leq C \int_{g^{-1}\left(\mathcal{K}^{*}\right)}\left|\frac{\nabla \tilde{v}(z)-\nabla\left(\tau_{h, k} \tilde{v}\right)(z)}{(h+k)^{\alpha}}\right|^{p} d z+C \int_{g^{-1}\left(\mathcal{K}^{*}\right)}\left|\frac{\nabla \tilde{v}(z)-\nabla\left(\tau_{-h, k} \tilde{v}\right)(z)}{(h+k)^{\alpha}}\right|^{p} d z .
\end{gathered}
$$

Summing all these estimates over the set of dual control volumes we find

$$
\left\|v^{\mathcal{T}}\right\|_{1+\alpha, p, \mathcal{T}}^{p} \leq C \int_{\widetilde{\Omega}}\left|\frac{\nabla \tilde{v}(z)-\nabla\left(\tau_{h, k} \tilde{v}\right)(z)}{(h+k)^{\alpha}}\right|^{p} d z+C \int_{\widetilde{\Omega}}\left|\frac{\nabla \tilde{v}(z)-\nabla\left(\tau_{-h, k} \tilde{v}\right)(z)}{(h+k)^{\alpha}}\right|^{p} d z .
$$

We just have now to bound the right-hand side above in terms of the norms of $v$ on the original domain $\Omega$. To this end, we compute

$$
\tau_{h, k} \partial_{\tilde{x}} \tilde{v}(\tilde{x}, \tilde{y})=g_{\mathrm{x}}^{\prime}\left(\tilde{x}+\frac{h}{2}\right) \partial_{x} v \circ \tau_{h, k} g(\tilde{x}, \tilde{y})
$$

so that

$$
\left|\tau_{h, k} \partial_{\tilde{x}} \tilde{v}(\tilde{z})-\partial_{\tilde{x}} \tilde{v}(\tilde{z})\right| \leq C h\left\|g_{\mathrm{x}}^{\prime \prime}\right\|_{\infty}\left|\partial_{x} v \circ g(\tilde{z})\right|+C\left\|g_{\mathrm{x}}^{\prime}\right\|_{\infty}\left|\partial_{x} v \circ \tau_{h, k} g(\tilde{z})-\partial_{x} v \circ g(\tilde{z})\right|
$$


Using the change of variables $g$ (whose Jacobian is bounded by $\operatorname{reg}(\mathcal{T})$ ), we obtain

$$
\begin{aligned}
\left\|v^{\mathcal{T}}\right\|_{1+\alpha, p, \mathcal{T}}^{p} \leq C \operatorname{size}(\mathcal{T})^{(1-\alpha) p} \int_{\Omega}|\nabla v(z)|^{p} d z+C \int_{\Omega}\left|\frac{\nabla v(z)-\nabla v\left(g \circ \tau_{h, k} \circ g^{-1}(z)\right)}{(h+k)^{\alpha}}\right|^{p} d z & +C \int_{\Omega}\left|\frac{\nabla v(z)-\nabla v\left(g \circ \tau_{-h, k} \circ g^{-1}(z)\right)}{(h+k)^{\alpha}}\right|^{p} d z .
\end{aligned}
$$

By definition of the vector fields $\theta_{\mathrm{x}}$ and $\theta_{\mathrm{y}}$ (given in Section 2), we clearly have

$$
e^{h \theta_{\mathrm{x}}} \circ e^{k \theta_{\mathrm{y}}}=g \circ \tau_{h, k} \circ g^{-1},
$$

so that we finally get that $\left\|\left|v^{\mathcal{T}}\right|\right\|_{1+\alpha, p, \mathcal{T}} \leq C \operatorname{size}(\mathcal{T})^{(1-\alpha)}\|v\|_{W^{1, p}}+C\|v\|_{\theta_{\mathbf{x}}, B_{\infty}^{1+\alpha, p}}+C\|v\|_{\theta_{\mathrm{y}}, B_{\infty}^{1+\alpha, p}}$.

\subsection{Discrete a priori estimate for the approximate solution. Proof of Proposition 3.4}

We reproduce, at the discrete level, the arguments of the proof of Theorem 1.1 given in [10]. First of all, just like in [2], it is possible to suppose that the boundary conditions are periodic (by extending the domain and the data by symmetry to a larger domain). Let us introduce the translation operators, for instance in the $x$ direction. We denote by $\tau \mathcal{K}$ (resp. $\tau \mathcal{K}^{*}$ ) the right neighbor of $\mathcal{K}$ (resp. $\mathcal{K}^{*}$ ) - taking into account the periodicity condition if necessary. In the same way we introduce $\tau^{-1} \mathcal{K}$ and $\tau^{-1} \mathcal{K}^{*}$ the left neighbors of $\mathcal{K}$ and $\mathcal{K}^{*}$. For any $v^{\mathcal{T}} \in \mathbb{R}^{\mathcal{T}}$ we introduce $\tau v^{\mathcal{T}} \in \mathbb{R}^{\mathcal{T}}$ and $\tau^{-1} v^{\mathcal{T}}$, defined by $\left(\tau v^{\mathcal{T}}\right)_{\mathcal{K}}=v_{\tau \mathcal{K}}$ and $\left(\tau^{-1} v^{\mathcal{T}}\right)_{\mathcal{K}}=v_{\tau^{-1}}$.

If we use $v^{\mathcal{T}} \in \mathbb{R}^{\mathcal{T}}$ as a discrete test function in (6), we get

$$
\sum_{\mathcal{K}^{*}} m\left(\mathcal{K}^{*}\right)\left(a_{\mathcal{K}^{*}} \circ T_{\mathcal{K}^{*}}\left(u^{\mathcal{T}}\right), T_{\mathcal{K}^{*}}\left(v^{\mathcal{T}}\right)\right)=\sum_{\mathcal{K}} m(\mathcal{K}) f_{\mathcal{K}} v_{\mathcal{K}}
$$

Replacing $v^{\mathcal{T}}$ by $\tau^{-1} v^{\mathcal{T}}$ in (16) we get

$$
\sum_{\mathcal{K}^{*}} m\left(\mathcal{K}^{*}\right)\left(a_{\mathcal{K}^{*}} \circ T_{\mathcal{K}^{*}}\left(u^{\mathcal{T}}\right), T_{\mathcal{K}^{*}}\left(\tau^{-1} v^{\mathcal{T}}\right)\right)=\sum_{\mathcal{K}} m(\mathcal{K}) f_{\mathcal{K}} v_{\tau^{-1} \mathcal{K}}
$$

Remarking that $T_{\tau \mathcal{K}^{*}}\left(v^{\mathcal{T}}\right)=T_{\mathcal{K}^{*}}\left(\tau v^{\mathcal{T}}\right)$ for any $\mathcal{K}^{*}$, we can change $\mathcal{K}^{*}$ into $\tau \mathcal{K}^{*}$ in the left-hand side and $\mathcal{K}$ into $\tau \mathcal{K}$ in the right-hand side. We obtain

$$
\sum_{\mathcal{K}^{*}} m\left(\tau \mathcal{K}^{*}\right)\left(a_{\tau \mathcal{K}^{*}} \circ T_{\mathcal{K}^{*}}\left(\tau u^{\mathcal{T}}\right), T_{\mathcal{K}^{*}}\left(v^{\mathcal{T}}\right)\right)=\sum_{\mathcal{K}} m(\tau \mathcal{K}) f_{\tau \mathcal{K}} v_{\mathcal{K}}
$$

Subtracting (16) from (17) we get

$$
\sum_{\mathcal{K}^{*}}\left(m\left(\tau \mathcal{K}^{*}\right) a_{\tau \mathcal{K}^{*}} \circ T_{\mathcal{K}^{*}}\left(\tau u^{\mathcal{T}}\right)-m\left(\mathcal{K}^{*}\right) a_{\mathcal{K}^{*}} \circ T_{\mathcal{K}^{*}}\left(u^{\mathcal{T}}\right), T_{\mathcal{K}^{*}}\left(v^{\mathcal{T}}\right)\right)=\sum_{\mathcal{K}}\left(m(\tau \mathcal{K}) f_{\tau \mathcal{K}}-m(\mathcal{K}) f_{\mathcal{K}}\right) v_{\mathcal{K}} .
$$

Introducing the operator

$$
D_{\mathcal{K}^{*}}: w \in \mathbb{R}^{4} \mapsto D_{\mathcal{K}^{*}}(w) \stackrel{\text { def }}{=} m\left(\tau \mathcal{K}^{*}\right) a_{\tau \mathcal{K}^{*}}(w)-m\left(\mathcal{K}^{*}\right) a_{\mathcal{K}^{*}}(w) \in \mathbb{R}^{4},
$$

we deduce

$$
\begin{aligned}
\sum_{\mathcal{K}^{*}} m\left(\mathcal{K}^{*}\right)\left(a_{\mathcal{K}^{*}} \circ T_{\mathcal{K}^{*}}\left(\tau u^{\mathcal{T}}\right)-a_{\mathcal{K}^{*}} \circ T_{\mathcal{K}^{*}}\left(u^{\mathcal{T}}\right), T_{\mathcal{K}^{*}}\left(v^{\mathcal{T}}\right)\right) \\
=\sum_{\mathcal{K}}\left(m(\tau \mathcal{K}) f_{\tau \mathcal{K}}-m(\mathcal{K}) f_{\mathcal{K}}\right) v_{\mathcal{K}}-\sum_{\mathcal{K}^{*}}\left(D_{\mathcal{K}^{*}}\left(T_{\mathcal{K}^{*}}\left(\tau u^{\mathcal{T}}\right)\right), T_{\mathcal{K}^{*}}\left(v^{\mathcal{T}}\right)\right) .
\end{aligned}
$$


We now choose $v^{\mathcal{T}}=\tau u^{\mathcal{T}}-u^{\mathcal{T}}$. It is shown in [2] that, for $p>2$, the left-hand side of (19) controls the discrete $W^{1, p}$ norm of the difference $\tau u^{\mathcal{T}}-u^{\mathcal{T}}$. Therefore (19) yields

$$
\frac{1}{C}\left\|\tau u^{\mathcal{T}}-u^{\mathcal{T}}\right\|_{1, p, \mathcal{T}}^{p} \leq\left|\sum_{\mathcal{K}}\left(m(\tau \mathcal{K}) f_{\tau \mathcal{K}}-m(\mathcal{K}) f_{\mathcal{K}}\right)\left(u_{\tau \mathcal{K}}-u_{\mathcal{K}}\right)\right|+\sum_{\mathcal{K}^{*}}\left|\left(D_{\mathcal{K}^{*}}\left(T_{\mathcal{K}^{*}}\left(\tau u^{\mathcal{T}}\right)\right), T_{\mathcal{K}^{*}}\left(\tau u^{\mathcal{T}}-u^{\mathcal{T}}\right)\right)\right|
$$

The first term in the right-hand side of (20) is estimated, as in [2], as follows:

$$
\left|\sum_{\mathcal{K}}\left(m(\tau \mathcal{K}) f_{\tau \mathcal{K}}-m(\mathcal{K}) f_{\mathcal{K}}\right)\left(u_{\tau \mathcal{K}}-u_{\mathcal{K}}\right)\right|=\left|\sum_{\mathcal{K}} m(\mathcal{K}) f_{\mathcal{K}}\left[\left(u_{\tau \mathcal{K}}-u_{\mathcal{K}}\right)-\left(u_{\mathcal{K}}-u_{\tau^{-1} \mathcal{K}}\right)\right]\right| \leq C h\|f\|_{L^{p^{\prime}}}\left\|\tau u^{\mathcal{T}}-u^{\mathcal{T}}\right\|_{1, p, \mathcal{T}} .
$$

In order to evaluate the second term in (20), which is new since it comes from the non-uniformity of the meshes, we need to estimate $D_{\mathcal{K}^{*}}(w)$ defined in (18).

Lemma 4.1. The following properties hold:

$$
\begin{gathered}
\left|\left(B_{\tau \mathcal{K}^{*}} v, w\right)-\left(B_{\mathcal{K}^{*}} v, w\right)\right| \leq C \operatorname{size}(\mathcal{T})\left|B_{\mathcal{K}^{*}}^{\frac{1}{2}} v \| B_{\mathcal{K}^{*}}^{\frac{1}{2}} w\right|, \quad \forall v, w \in \mathbb{R}^{4}, \\
\left\|B_{\tau \mathcal{K}^{*}}-B_{\mathcal{K}^{*}}\right\| \leq C \operatorname{size}(\mathcal{T})\left\|B_{\mathcal{K}^{*}}\right\|, \\
\left|B_{\tau \mathcal{K}^{*}}^{\frac{1}{2}} v-B_{\mathcal{K}^{*}}^{\frac{1}{2}} v\right| \leq C \operatorname{size}(\mathcal{T})\left|B_{\mathcal{K}^{*}}^{\frac{1}{2}} v\right|, \quad \forall v \in \mathbb{R}^{4}
\end{gathered}
$$

Proof. The first estimate follows immediately from (3), the explicit formula (8), Definition 2.1 and (9). Notice that we use here the fact that $B_{\mathcal{K}^{*}}$ and $B_{\tau \mathcal{K}^{*}}$ have the same kernel $\operatorname{Span}\left\{(1,1,1,1)^{t}\right\}$, which is denoted by $E_{0}$ in the sequel. The second estimate is a straightforward consequence of the first one.

In order to prove (23), we use the following Lipschitz property of the square root operator over the set of symmetric definite positive (s.d.p.) matrices:

For any $\lambda_{1}, \lambda_{2}>0$, there exists $M_{\lambda_{1}, \lambda_{2}}>0$ such that

$$
\left\|A^{\frac{1}{2}}-B^{\frac{1}{2}}\right\| \leq M_{\lambda_{1}, \lambda_{2}}\|A-B\|, \quad \forall A, B \text { s.d.p. such that } \operatorname{Sp}(A) \cup \operatorname{Sp}(B) \subset\left[\lambda_{1}, \lambda_{2}\right]
$$

This property is a consequence of the Cauchy integral formula. We established during the proof of (9) (see [1, Lemma 2.8]) that the restriction of $B_{\mathcal{K}^{*}}$ to $E_{0}^{\perp}$ is definite positive and its spectrum $\operatorname{lies}$ in $\left[\frac{1}{C m\left(\mathcal{K}^{*}\right)}, \frac{C}{m\left(\mathcal{K}^{*}\right)}\right]$ where $C$ depends on $\operatorname{reg}(\mathcal{T})$ and $\operatorname{adm}(\mathcal{T}, \Xi)$. Hence, there exists $\lambda_{1}, \lambda_{2}>0$ depending on $\operatorname{reg}(\mathcal{T}) \operatorname{and} \operatorname{adm}(\mathcal{T}, \Xi)$ such that the spectrums of the restrictions to $E_{0}^{\perp}$ of the matrices $\left(\operatorname{size}(\mathcal{T})^{2} B_{\mathcal{K}^{*}}\right)_{\mathcal{K}^{*}}$ are all included in $\left[\lambda_{1}, \lambda_{2}\right]$. Hence, (23) follows from (24).

Using the estimates given in Lemma 4.1 we can now bound $D_{\mathcal{K}^{*}}$ as follows:

Lemma 4.2. There exists $C>0$ such that

$$
\left|\left(D_{\mathcal{K}^{*}}(v), w\right)\right| \leq C \operatorname{size}(\mathcal{T}) m\left(\mathcal{K}^{*}\right)\left(\left|B_{\mathcal{K}^{*}}^{\frac{1}{2}} v\right|+\left|B_{\tau \mathcal{K}^{*}}^{\frac{1}{2}} v\right|\right)^{p-1}\left(\left|B_{\mathcal{K}^{*}}^{\frac{1}{2}} w\right|+\left|B_{\tau \mathcal{K}^{*}}^{\frac{1}{2}} w\right|\right), \quad \forall \mathcal{K}^{*} \in \mathcal{T}^{*}, \forall v, w \in \mathbb{R}^{4}
$$

Proof. We have

$$
\left|\left(D_{\mathcal{K}^{*}}(v), w\right)\right| \leq\left|m\left(\tau \mathcal{K}^{*}\right)-m\left(\mathcal{K}^{*}\right)\right|\left|\left(a_{\tau \mathcal{K}^{*}}(v), w\right)\right|+m\left(\mathcal{K}^{*}\right)\left|\left(a_{\tau \mathcal{K}^{*}}(v)-a_{\mathcal{K}^{*}}(v), w\right)\right| .
$$

Note that $\left|m\left(\tau \mathcal{K}^{*}\right)-m\left(\mathcal{K}^{*}\right)\right| \leq C \operatorname{size}(\mathcal{T}) m\left(\mathcal{K}^{*}\right)$, with $C$ controlled by $\operatorname{reg}(\mathcal{T})$. Hence, by definition of $a_{\mathcal{K}^{*}}$, the first term in the right-hand side of $(25)$ is bounded by $C \operatorname{size}(\mathcal{T}) m\left(\mathcal{K}^{*}\right)\left|B_{\tau \mathcal{K}^{*}}^{\frac{1}{2}} v\right|^{p-1}\left|B_{\tau \mathcal{K}^{*}}^{\frac{1}{2}} w\right|$. 
It is well known that there exists $M>0$ depending only on $p \geq 2$, such that for any $n \geq 1$

$$
\left.|| \xi\right|^{p-2} \xi-|\eta|^{p-2} \eta|\leq M| \xi-\eta \mid\left(|\xi|^{p-2}+|\eta|^{p-2}\right), \quad \forall \xi, \eta \in \mathbb{R}^{n}
$$

Hence, using (23), we can control the second term in the right hand side of (25) (denoted by $T$ ) by

$$
\begin{aligned}
T & \leq m\left(\mathcal{K}^{*}\right)\left|\left(\left|B_{\tau \mathcal{K}^{*}}^{\frac{1}{2}} v\right|^{p-2} B_{\tau \mathcal{K}^{*}}^{\frac{1}{2}} v-\left|B_{\mathcal{K}^{*}}^{\frac{1}{2}} v\right|^{p-2} B_{\mathcal{K}^{*}}^{\frac{1}{2}} v, B_{\mathcal{K}^{*}}^{\frac{1}{2}} w\right)\right|+m\left(\mathcal{K}^{*}\right)\left|B_{\tau \mathcal{K}^{*}}^{\frac{1}{2}} v\right|^{p-2}\left|\left(B_{\tau \mathcal{K}^{*}}^{\frac{1}{2}} v, B_{\tau \mathcal{K}^{*}}^{\frac{1}{2}} w-B_{\mathcal{K}^{*}}^{\frac{1}{2}} w\right)\right| \\
& \leq C m\left(\mathcal{K}^{*}\right)\left|B_{\mathcal{K}^{*}}^{\frac{1}{2}} v-B_{\tau \mathcal{K}^{*}}^{\frac{1}{2}} v\right|\left(\left|B_{\mathcal{K}^{*}}^{\frac{1}{2}} v\right|+\left|B_{\tau \mathcal{K}^{*}}^{\frac{1}{2}} v\right|\right)^{p-2}\left|B_{\mathcal{K}^{*}}^{\frac{1}{2}} w\right|+C m\left(\mathcal{K}^{*}\right)\left|B_{\tau \mathcal{K}^{*}}^{\frac{1}{2}} v\right|^{p-1}\left|B_{\mathcal{K}^{*}}^{\frac{1}{2}} w-B_{\tau \mathcal{K}^{*}}^{\frac{1}{2}} w\right| \\
& \leq C \operatorname{size}(\mathcal{T}) m\left(\mathcal{K}^{*}\right)\left|B_{\mathcal{K}^{*}}^{\frac{1}{2}} v\right|\left(\left|B_{\mathcal{K}^{*}}^{\frac{1}{2}} v\right|+\left|B_{\tau \mathcal{K}^{*}}^{\frac{1}{2}} v\right|\right)^{p-2}\left(\left|B_{\mathcal{K}^{*}}^{\frac{1}{2}} w\right|+\left|B_{\tau \mathcal{K}^{*}}^{\frac{1}{2}} w\right|\right),
\end{aligned}
$$

and the claim is proved.

Finally, collecting all these estimates in (20) and using (9) and the Poincaré inequality, we find

$$
\left\|\tau u^{\mathcal{T}}-u^{\mathcal{T}}\right\|_{1, p, \mathcal{T}}^{p} \leq C \operatorname{size}(\mathcal{T})\|f\|_{L^{p^{\prime}}}\left\|\tau u^{\mathcal{T}}-u^{\mathcal{T}}\right\|_{1, p, \mathcal{T}}+C \operatorname{size}(\mathcal{T})\left\|u^{\mathcal{T}}\right\|_{1, p, \mathcal{T}}^{p-1}\left\|\tau u^{\mathcal{T}}-u^{\mathcal{T}}\right\|_{1, p, \mathcal{T}}
$$

Using (11), we finally deduce $\frac{1}{\operatorname{size}(\mathcal{T})^{\alpha}}\left\|\tau u^{\mathcal{T}}-u^{\mathcal{T}}\right\|_{1, p, \mathcal{T}} \leq C\|f\|_{L^{p^{\prime}}}^{\frac{1}{p-1}}$ and we recover the discrete Besov estimate by noting that, $\tau$ being the translation in the $x$ direction, the discrete function $\frac{\delta_{2}^{\mathcal{K}^{*}}\left(\tau u^{\mathcal{T}}-u^{\mathcal{T}}\right)}{\operatorname{size}(\mathcal{T})^{\alpha}}$ is exactly made of differential quotients like in the definition (5) of the discrete Besov norm.

The second author wishes to thank S. Labbé for giving him the opportunity to give a talk in the Groupe de Travail Numérique of the university Paris XI.

\section{REFERENCES}

[1] B. Andreianov, F. Boyer and F. Hubert, Finite volume schemes for the p-laplacian on cartesian meshes, $M^{2} A N$ Math. Model. Numer. Anal., 38, n 6, pp. 931-960, 2004.

[2] B. Andreinnov, F. Boyer and F. Hubert, Besov regularity and new error estimates for finite volume approximations of the p-laplacian, Numer. Math., 100, n 4, pp. 565-592, 2005.

[3] B. Andreianov, F. Boyer and F. Hubert, On the finite volume approximation of regular solutions of the p-laplacian, to appear in IMA J. Numer. Anal., http://dx.doi.org/doi:10.1093/imanum/dri047, 2006.

[4] B. Andreianov, F. Boyer and F. Hubert, Discrete duality finite volume schemes for Leray-Lions type elliptic problems on general 2D meshes, Numerical Methods for PDEs, to appear, 2006.

[5] B. Andreianov, M. Gutnic and P. Wittbold, Convergence of finite volume approximations for a nonlinear elliptic-parabolic problem : a "continuous" approach, SIAM J. on Numer. Anal., 42 no 1, pp. 228-251, 2004.

[6] S. Chow, Finite element error estimates for non-linear elliptic equations of monotone type, Numer. Math. 54, pp. 373-393, 1989.

[7] J. Droniou, Finite volume approximations for fully non-linear elliptic equations in divergence form, submitted, http://hal.ccsd.cnrs.fr/ccsd-00009614, 2005.

[8] T. Runst And W. Sickel, Sobolev spaces of fractional order, Nemytskij operators and nonlinear partial differential equations, De Gruyter series in nonlinear analysis and applications, De Gruyter, 1996.

[9] J. Simon, Caractérisation d'espaces fonctionnels, Bollettino U. M.I. 15-B , pp. 687-714, 1978.

[10] J. Simon, Régularité de la solution d'un problème aux limites non linéaires, Ann. Fac. Sciences Toulouse 3, pp. 247-274, 1981.

[11] H. Triebel, Interpolation theory, function spaces, differential operators, North-Holland, Amsterdam, 1978.

[12] V.B. Tyukthin, The rate of convergence of approximation methods for solving one-sided variational problems. I, Teoret. Mat. Fiz. 51 n $^{\circ}$ 2, pp 111-113, 121, 1982. 